\title{
Comparison of various histochemical staining methods for identification of helicobacter pylori
}

\author{
Gowsik K. ${ }^{1}$, Archana V. ${ }^{2}$ \\ ${ }^{1}$ Dr. Kanimozhi Gowsik, Assistant Professor, Aarupadai Veedu Medical College, ${ }^{2}$ Dr. Archana V., Assistant Professor; \\ Dhanalakshmi Srinivasan Medical College and Hospital, Tamil Nadu, India.
}

Corresponding Author: Dr. Archana V., Assistant Professor, Dhanalakshmi Srinivasan Medical College and Hospital, Tamil Nadu, India. E-mail: archanasureshraj@gmail.com

\begin{abstract}
Introduction: Helicobacter pylori infection plays a key role in the development of gastritis, Gastric ulcer and Gastric malignancy. More than half the world's population is infected with this organism. The infection is more common in developing countries due to poor sanitation, overcrowding and low economic status. In view of this pathogenetic importance, diagnosis of $H$. Pylori is very important to institute eradication treatment. Various techniques are available for the detection of H. Pylori including serology, rapid urease test,13C-breath test, antigen detection in stool, histological examination and IHC. Aim: This study was conducted to compare various histochemical staining methods for identification of helicobacter pylori in endoscopic biopsy taken for gastritis. Materials and Methods: A total of 68 cases were included in this study over a period of six months. Slides were stained with 5 different histochemical stains. Sensitivity, Specificity and positive predictive value was calculated. As per literature modified giemsa was considered as standard and the findings from other stains were compared with it. Results: Out of 68 cases of gastric biopsy diagnosed of gastritis, 23 cases were positive for H. Pylori with Modified Giemsa, 17 with H\&E, 23 with Toluidine blue, 21 with Acridine orange, 14 with Alcian blue. Conclusion: Histopathological examination is the gold standard method for identification. whichever stains used careful examination for the organism is essential. All the staining methods were easy to perform and cheap. Modified Giemsa, H\&E and Acridine orange are more reliable.
\end{abstract}

Keyword: Gastric ulcer, Gastric malignancy, Eradication treatment

\section{Introduction}

Helicobacter Pylori infection plays a key role in the development of gastritis, gastric ulcer and gastric malignancy. More than half the world's population is infected with this organism [1]. The infection is more common in developing countries due to poor sanitation, overcrowding and low economic status [2].

In 1875, German scientists documented helical shaped bacteria from stomach lining, but they were not able to culture and it was forgotten. The organism was rediscovered by Dr. Barry. J. Marshall and Dr. J. Robin Warren in the year 1983, followed by cascade of reports regarding their association with chronic gastritis, peptic ulcers of stomach, duodenum, adenocarcinoma and lymphoma [3, 4]. H. pylori produces urease which converts urea to $\mathrm{CO}_{2}$ and $\mathrm{NH}_{3}$, which in turn buffers the gastric acid and elevates the $\mathrm{pH}$ to 5.5 which will be suitable for the survival of the bacteria. In antral region

Manuscript received: $25^{\text {th }}$ August 2019

Reviewed: $6^{\text {th }}$ September 2019

Author Corrected: $11^{\text {th }}$ September 2019

Accepted for Publication: $16^{\text {th }}$ September 2019 the optimal $\mathrm{pH}$ is obtained easily so the anaerobic bacteria colonize in higher concentration during acute infection.

The bacteria produce reductases which can convert nitrate in the food to nitrite which in turn can react with amines, amides and urease to produce carcinogenic Nnitroso compounds $[5,6]$.

Genetically the organism is hetrogenous and all strains does not cause malignancy. The organisms which are cag and VacA positive strain can cause gastric carcinoma. The former causes production of interleukin 8 (IL8) through nuclear factor kappa B pathway and the latter produces vacuoalating cytotoxin. Increased production of IL-8 causes inflammation which in turn leads to atrophic gastritis and diffuse antral gastritis. Atrophic gastritis leads to intestinal metaplasia and latter leading to intestinal adenocarcinoma. In diffuse antral gastritis lymphoid follicles and germinal centres are seen in stroma. So formation of MALT as an 


\section{Original Research Article}

immune response to $H$. pylori infection may be an essential precursor to MALT lymphoma.

In view of this pathogenetic importance, diagnosis of $H$. Pylori is very important to institute eradication treatment [7].

Various techniques are available for the detection of $H$. Pylori including serology, rapid urease test, 13C-breath test, antigen detection in stool, histological examination and $\mathrm{IHC}[8,9]$.

Histological identification of the bacilli by various staining methods are in use for identification of the organism. The present study was conducted to compare various histochemical staining methods for identification of H. Pylori in endoscopic biopsies.

\section{Materials and Methods}

Type of study and study period: This was a prospective observational study in a rural tertiary care hospital over six months period.
Ethical approval: The institutional medical ethics committee approved this study.

Inclusion criteria: All cases of gastritis in the sixmonth period.

Exclusion criteria: Biopies that was too less for demonstration of five stains.

Study design: All endoscopic gastric mucosal biopsies taken from different sites were fixed in $10 \%$ buffered formalin. After overnight fixation in formalin, processing was done, followed by paraffin embedding and section cutting in rotary microtome. Serial sections of 3-5 $\mu \mathrm{m}$ thickness were made \& stained with Modified Giemsa, Haematoxylin \& Eosin, Alcian Blue, Toluidine Blue and Acridine Orange. As per literature Modified Giemsa was considered as standard and the findings of other stains were compared with Modified Giemsa.

Histopathological study of gastric biopsies was done. These findings were being analyzed and they were compared with the findings of other authors.

\section{Results}

Of the 68 cases studied 23 were positive for H.Pylori infection considering histopathology section stained by Modified giemsa as gold standard (Table 1).

Table-1: Results of different staining techniques

\begin{tabular}{|c|c|c|}
\hline Different Staining Techniques & H.pylori positive & H.pylori Negative \\
\hline Modified Giemsa & 23 & 45 \\
\hline Haematoxylin and Eosin & 17 & 51 \\
\hline Toludine blue & 23 & 45 \\
\hline Alcian blue & 14 & 54 \\
\hline Acridine orange & 21 & 47 \\
\hline
\end{tabular}

The results of other staining techniques were compared with it. Sensitivity, specificity, accuracy, positive predictive value and negative predictive value for different stains were calculated based on this (Table 2).

Table-2: Sensitivity, Specificity, Accuracy, Positive predictive value and Negative predictive value for Different Stains

\begin{tabular}{|l|c|c|c|c|}
\hline & H and E & AB & TB & AO \\
\hline No. of positive cases & 17 & 14 & 23 & 21 \\
\hline True positive (a) & 16 & 10 & 16 & 21 \\
\hline false positive (b) & 1 & 4 & 7 & 0 \\
\hline False negative (c) & 7 & 13 & 7 & 2 \\
\hline True negative (d) & 44 & 41 & 38 & 45 \\
\hline Sensitivity a/(a+c)*100 & 69.56 & 43.47 & 69.56 & 91.30 \\
\hline Specificity d/(c+d)*100 & 97.77 & 91.11 & 84.4 & 95.74 \\
\hline Accuracy ( a+d/a+b+c+d)*100 & 88.23 & 75 & 79.41 & 97.05 \\
\hline Positive predictive value a/a+b *100 & 94.11 & 71.42 & 69.56 & 100 \\
\hline Negative predictive value d/d+c* 100 & 86.27 & 75.92 & 84.44 & 95.74 \\
\hline
\end{tabular}




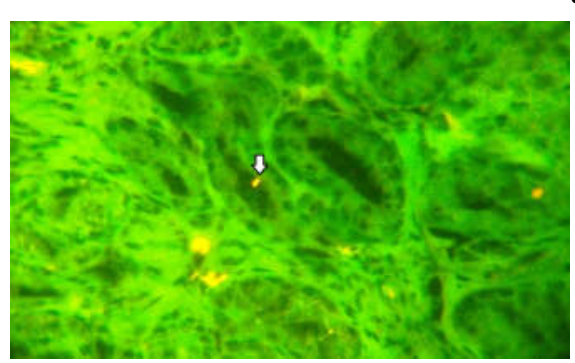

\section{Original Research Article}

Figure-1: Demonstration of H. pylori by Acridine Orange Stain in a fluorescence Microscope;

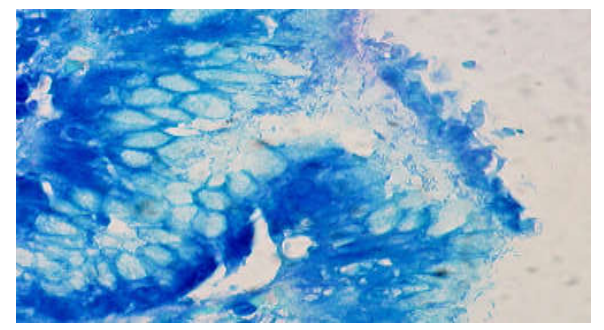

Figure-2: Demonstration of $\boldsymbol{H}$. pylori by Alcian blue Stain

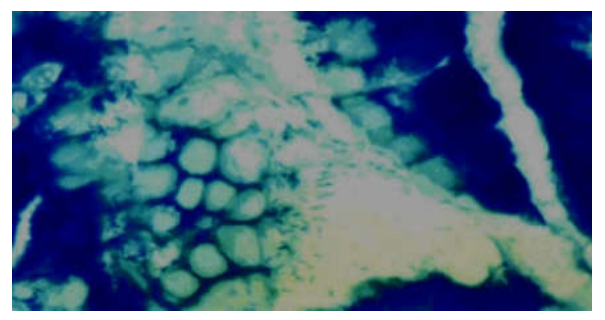

Figure-3: Demonstration of $\boldsymbol{H}$. pylori by Toluidine blue Stain

\section{Discussion}

H. Pylori detection can be done by various techniques, but the aim of the research is to identify a technique which is cost effective, rapid and accurate. Number of bacteria in the specimen determines the sensitivity of the test. Every test in the hands of experienced has good sensitivity and specificity [10]. In the present study cases positive with acridine orange showed $97 \%$ accuracy which is same as Haqqani MT, LangdaleBrown et al [10], they conducted two different studies in 1998 and have mentioned that Acridine Orange is not specific but $100 \%$ accurate and study by Rotimi $\mathrm{O}$ et al [7] in 2000 also suggested the same. It is easy, cheap and simple procedure to perform but most of the labs do not have fluorescence attachment and Haqqani also criticized about the same and said "The only disadvantage of the acridine orange stain is that the microscope needs a fluorescent attachment. In the present study alcian blue stain showed less sensitivity when compared to other study [11]. Kaur et al [12] in his study compared different techniques and found that alcian blue was a bit time consuming procedure but the results were quite satisfactory.
The organism did not appear contrasting to the surrounding tissue and that appeared to be the reason for false negativity. Kaur et al [12] in his study observed Toluidine Blue stain was cheap and easily applicable and consumed only 4 minutes. However, its sensitivity and specificity were less when compared with MG and it was the same in the present study where sensitivity was $69.5 \%$ and specificity was $84.4 \%$. The inter observer agreement was relatively low for TB stain. Previous studies $[12,13]$ et al found that $\mathrm{H} \& \mathrm{E}$ stain was cost effective to use as it is routinely performed for the evaluation of gastric biopsies.

Wilkins [12] in her study said that increase in staining time of haematoxylin can give good results. Sensitivity $(69.5 \%)$ and accuracy $(88.23 \%)$ was low in the present study which was similar to their studies, due to lack of contrast between the bacteria and the surrounding tissues H\&E in combination with a special stain may be a cost effective way of demonstrating the bacteria [14, 15]. The main limitation of the present study is smaller sample size. 


\section{Original Research Article}

\section{Conclusion}

All the staining techniques are easy to perform and cheap., Modified Giemsa, H \& E, Acridine orange and Alcian blue stains are reliable. Toludine blue was less specific and had less sensitivity than other stains.

\section{What the study adds to the existing knowledge?}

Use of acridine orange is limited due to presence of fluorescence attachment to the microscope. But patience and spending more time in searching for the bacilli would give good results.

\section{Author's contribution}

1. Dr. Kanimozhi Gowsik: Preparation of stains, Collection of samples, review of slides.

2. Dr. Archana V.: Writing of the article, Collection of samples, review of slides.

Findings: Nil; Conflict of Interest: None initiated Permission from IRB: Yes

\section{References}

1. World gastroenterology organization global guideline: Helicobacter pylori in developing countries. J Dig Dis 2011;12 (5):319-326. doi: 10.1111/j.17512980.2011.00529. x.

2. Mills AS, Contos MJ. The Stomach. In: Silverberg SG (ed). Silverberg's Principles and Practice of Surgical Pathology and Cytopathology. $4^{\text {th }}$ edition. Churchill Livingstone, 2005; 1321-1372.

3. Marshall B. Helicobacter pylori--a Nobel pursuit? Can J Gastroenterol. 2008; 22 (11):895-896. doi:10. $1155 / 2008 / 459810$

4. Rosai J, Chapter 11. Gastrointestinal tract. Rosai and Ackerman's Surgical Pathology, $9^{\text {th }}$ ed. Elsevier, Mosby.2004; 1:615-871.

5. Fenoglio-Preiser C, Carneiro F, Correa P, Guilford P, Lambert R, Megraud F, et al. Gastric carcinoma. Pathology and genetics of tumours of the digestive system. World Health Organization Classification of Tumors.2000;1:35-52.
6. Rotimi O, Cairns A, Gray S, Moayyedi P, Dixon MF. Histological identification of Helicobacter pylori: comparison of staining methods. J Clin Pathol. 2000;53(10):756-759. doi: 10.1136/jep.53.10.756

7. Yakoob J, Jafri W, Abid S, Jafri N, Abbas Z, Hamid $\mathrm{S}$, et al. Role of rapid urease test and histopathology in the diagnosis of Helicobacter pylori infection in a developing country. BMC Gastroenterol. 2005; 5:38. doi: 10.1186/1471-230X-5-38

8. Mesher AL. Junqueira's Basic Histology. Chapter 15. Digestive Tract. $12^{\text {th }}$ ed. The McGraw-Hill Companies. 2010; 312-346.

9. Loffeld RJ, Stobberingh E, Arends JW. A review of diagnostic techniques for Helicobacter pylori infection. Digestive Diseases. 1993;11(3):173-180.

10. Haqqani MT, Langdale-Brown B. Campylobacter pylori--acridine orange stain and ultraviolet fluorescence. Histopathol. 1988;12(4):456-457. doi: 10.1111/j.1365-2559. 1988.tb01964.x

11. Sandra Wilkins, AS, HT(ASCP) Helicobacter pylori, Michigan Institute of Urology.

12. Kaur G, Madhavan M, Basri AH, Sain AH, Hussain MS, Yatiban MK, Naing NN. Rapid diagnosis of Helicobacter pylori infection in gastric imprint smears. Southeast Asian J Trop Med Public Health. 2004;35(3):676-680.

13. Graham DY. Campylobacter pylori and peptic ulcer disease. Gastroenterol. 1989;96(2 Pt 2 Suppl):615-625. doi: 10.1016/s0016-5085(89)80057-5.

14. Misra V, Misra SP, Dwivedi M, Gupta SC. The Loeffler's methylene blue stain: an inexpensive and rapid method for detection of Helicobacter pylori. J Gastroenterol Hepatol. 1994;9 (5):512-513. doi: 10.1111/j.1440-1746. 1994.tb01283.x

15. Ahluwalia C, Jain M, Mehta G, Kumar N. Comparison of endoscopic brush cytology with biopsy for detection of Helicobacter pylori in patients with gastroduodenal diseases. Indian J Pathol Microbiol. 2001;44(3):283-288.

\section{How to cite this article?}

Gowsik K., Archana V. Comparison of various histochemical staining methods for identification of helicobacter pylori. Trop J Path Micro 2019;5(9):692-695.doi:10.17511/jopm.2019.i09.12. 